\title{
CINÉTICA DE ADSORÇÃO DE CORANTES ALIMENTÍCIOS EM SISTEMA BINÁRIO POR QUITOSANA COM E SEM MODIFICAÇÃO
}

\author{
J. O. GONÇALVES ${ }^{1 *}$, K. A. SILVA ${ }^{1}$, B. S. FARIAS, L. A. A. PINTO \\ ${ }^{1}$ Universidade Federal do Rio Grande, Escola Química e Alimentos \\ "e-mail: janaina_sde@hotmail.com
}

\begin{abstract}
RESUMO
O estudo da modificação da quitosana vem ganhando destaque na literatura, devido à possibilidade de melhorar a sua estabilidade química, aumentar a área superficial, facilitar a separação, dentre outras. A utilização da cianoguanidina na modificação, além de ser pouco investigada, torna o processo de adsorção mais barato devido ao seu custo benefício. O presente trabalho teve como objetivo verificar o comportamento cinético da adsorção dos corantes alimentícios por quitosana sem e com modificação. A quitosana foi obtida, após modificada com cianoguanidina e caracterizada em relação a área superficial específica (MEV) e grupamentos funcionais (FT-IR). Estudos cinéticos de adsorção foram realizados utilizando os corantes azul indigotina e amarelo tartrazina em sistema binário por diferentes quitosanas. Os modelos cinéticos pseudo-primeira ordem, pseudo-segunda ordem e Avrami foram ajustados aos dados experimentais. A quitosana modificada apresentou uma superfície mais heterogênea e com protuberâncias quando comparada com a quitosana sem modificação. O modelo de Avrami foi o que apresentou melhor ajuste aos dados experimentais $\left(R^{2}>0,95\right.$ e EMR $\left.<10,0 \%\right)$. As capacidades máximas de adsorção para o amarelo tartrazina e azul indigotina, foram respectivamente, de $225 \mathrm{mg} \mathrm{g}^{-1}$ e $205 \mathrm{mg} \mathrm{g}^{-1}$, tanto para as quitosanas sem e com modificação.
\end{abstract}

\section{INTRODUÇÃO}

Os corantes são moléculas recalcitrantes e muito estáveis, apresentam estruturas aromáticas e complexas, assim os tornando difíceis de serem biodegradados (GUPTA; SUHAS, 2009). Ao serem descartados no meio ambiente, os efluentes coloridos são considerados altamente tóxicos para a vida aquática, pois afetam os processos fotossintéticos e simbióticos, devido a estes interferirem na passagem da luz solar e assim reduzirem a capacidade de reoxigenação da água (SRINIVASAN; VIRARAGHAVAN, 2010). Portanto, é relevante o estudo de técnicas que auxiliem na remoção destes corantes em efluentes.

A operação de adsorção, em baixas concentrações, se torna mais viável quando comparada aos métodos convencionais (oxidação, separação por membranas e foto catálise) devido ao baixo custo, alta eficiência e simples operação (SALLEH et al., 2011; DEMIRBAS, 2009). A escolha do material adsorvente é de grande importância para o custo do processo do tratamento de efluentes coloridos (KUNZ et al., 2002). Dentre os diferentes tipos de adsorventes, a quitosana se destaca por ser versátil, apresentar alta eficiência, cinética rápida, disponibilidade e 
custo benefício (SRINIVASAN e VIRARAGHAVAN, 2010).

A quitosana é um bipolímero biodegradável que apresenta em sua estrutura grupos funcionais amino e hidroxila com alto potencial para interação com corantes (WAN NGAH; TEONG; HANAFIAH, 2011). Além de ser versátil, a quitosana pode ser modificada, tanto na sua forma física como em sua estrutura química. A modificação química proporciona uma maior estabilidade do biopolímero em meio ácido, possibilidade de incrementar o desempenho na adsorção e reduzir custos do adsorvente (RINAUDO, 2006; FU; WANG, 2011; ZHOU et al., 2014).

Um tipo de agente reticulante utilizado na modificação química da quitosana é a cianoguanidina. Um sólido cristalino incolor, de baixa massa molar, sendo um componente de baixo custo (DRONSKOWSKI; HUI-LIU, 2003; MA et al., 2011). A literatura apresenta poucos estudos voltados para a adsorção de corantes alimentícios por quitosana modificada com cianoguanidina, apesar desta, manter a estabilidade do biopolímero em meio ácido e também reduzir o custo do adsorvente (WANG et al., 2013).

O presente trabalho teve como objetivo avaliar o comportamento cinético da adsorção dos corantes alimentícios (azul indigotina e amarelo tartrazina) em sistema binário por quitosana com e sem modificação. A quitosana foi modificada com cianoguanidina, e os adsorventes foram caracterizados utilizando as técnicas de análise de infravermelho com transformada de Fourier (FT-IR) e microscopia eletrônica de varredura (MEV). Foram ajustados os modelos de pseudo-primeira ordem, pseudosegunda ordem e Avrami para correlacionar os dados experimentais cinéticos.

\section{MATERIAL E MÉTODOS}

\subsection{Adsorbato}

Neste trabalho, foram utilizados os dois corantes alimentícios, azul indigotina e amarelo tartrazina. Os corantes foram fornecidos pela indústria Duas Rodas Ind. (Brasil), com grau de pureza de 85\%. A Tabela 1 apresenta as características dos corantes.

Tabela 1 - Características dos corantes alimentícios.

\begin{tabular}{|c|c|c|}
\hline Cora & $\begin{array}{l}\text { Índice } \\
\text { de cor } \\
\text { (C.I.) }\end{array}$ & $\begin{array}{l}\text { Classe } \\
\text { química }\end{array}$ \\
\hline
\end{tabular}

Azul

indigotina 73015 Indigóide 466,35

Amarelo

tartrazina $19140 \quad$ Azo

534,4

As estruturas químicas dos corantes alimentícios estão ilustradas na Figura 1.

Figura 1 - Estruturas químicas dos corantes: (a) azul indigotina e (b) amarelo tartrazina

(a)<smiles>O=C1/C(=C2\Nc3ccc(S(=O)(=O)[O-])cc3C2=O)Nc2ccc(S(=O)(=O)[O-])cc21</smiles>

(b)

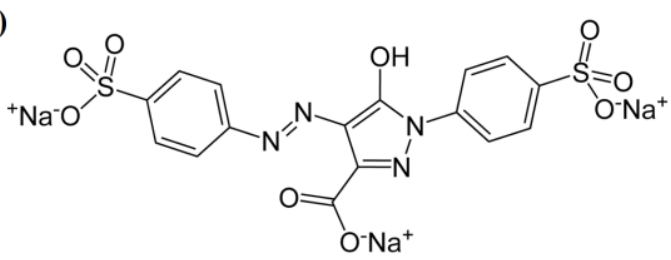

Fonte: Autores (2015)

O comprimento de onda máximo do corante azul indigotina é de $610 \mathrm{~nm}$ e do amarelo tartrazina é $425 \mathrm{~nm}$. 
A partir da Figura 1 pode-se verificar a presença dos grupamentos $\left(\mathrm{SO}^{3}\right)^{-}$presentes nas estruturas dos dois corantes, caracterizando-os como corantes aniônicos (GUPTA; SUHAS, 2009).

\subsection{Adsorventes}

Os adsorventes utilizados foram quitosana e quitosana modificada com cianoguanidina. A quitosana foi obtida a partir de rejeitos de camarão (Penaeus brasiliensis), fornecidos por indústrias de pescado da cidade de Rio Grande/RS.

Os rejeitos passaram por três etapas principais: desmineralização (ácido clorídrico 2,5 mL/100 mL); desproteinização (hidróxido de sódio $5 \mathrm{~g} / 100 \mathrm{~mL}$ ) desodorização e despigmentação (hipoclorito de sódio 0,36 $\mathrm{mL} / 100 \mathrm{~mL}$ ). Após, o material foi seco por 4 $\mathrm{h}$ à $80^{\circ} \mathrm{C}$, obtendo-se a quitina (MOURA et. al., 2011).

A quitina foi desacetilada em um reator de aço inoxidável com aquecimento e agitação mecânica, contendo $3 \mathrm{~L}$ de solução de $\mathrm{NaOH}$ (421 $\mathrm{g} \mathrm{L}^{-1}$ ) sob agitação de $50 \mathrm{rpm}$, à temperatura de $130^{\circ} \mathrm{C}$ por 90 min (WESKA et al., 2007).

A quitosana obtida foi purificada e seca em leito de jorro, apresentando diâmetro de $70 \mu \mathrm{m}$ (DOTTO et al., 2011).

\subsubsection{Modificação da quitosana com cianoguanidina \\ Para a modificação da quitosana,} foram utilizadas $1 \mathrm{~g}$ de quitosana (grau de desacetilação 95\%, massa molar de $120 \pm 4$ $\mathrm{kDa})$ dissolvida em $100 \mathrm{~mL}$ de ácido clorídrico $1 \%$ (v/v) sob agitação até completa dissolução. Após foram adicionados $0,53 \mathrm{~g}$ de cianoguanidina (Merck, 99,9\%), a temperatura da reação foi elevada para $90^{\circ} \mathrm{C} \mathrm{e}$ a solução foi agitada durante $3 \mathrm{~h}$. A solução de quitosana modificada com cianoguanidina foi deixada em repouso até que atingisse a temperatura ambiente, após foi seca em estufa à $40^{\circ} \mathrm{C}$ por $24 \mathrm{~h}$, assim obtendo-se o pó de quitosana modificada (WANG et al., 2013).

As amostras de quitosana antes e após a modificação foram caracterizadas por microscopia eletrônica de varredura (MEV) (Jeol, JSM-6060, Japão) e análise de infravermelho (FT-IR) (Prestige, 21210045, Japão), a fim de comprovar a modificação.

\subsection{Ensaios cinéticos}

Nos experimentos cinéticos de adsorção, foi preparada a solução com a mistura dos corantes apresentando uma concentração final de $100 \mathrm{mg} \mathrm{L} \mathrm{L}^{-1}$. Esta solução foi preparada através da diluição de $50 \mathrm{~mL}$ de corante amarelo tartrazina (1000 $\left.\mathrm{mg} \mathrm{\textrm {L } ^ { - 1 }}\right), 50 \mathrm{~mL}$ de corante azul indigotina (1000 $\mathrm{mg} \mathrm{L}^{-1}$ ) em água destilada. $\mathrm{O} \mathrm{pH}$ da solução foi corrigido $(\mathrm{pH}=3,0)$ através da adição de $100 \mathrm{~mL}$ tampão fosfato dissódico/ácido cítrico $0,1 \mathrm{~mol} \mathrm{~L}^{-1}$. Esta condição foi estabelecida em estudos anteriores. Então, $250 \mathrm{mg}$ de de quitosana modificada e sem modificação foram adicionadas em $1 \mathrm{~L}$ de solução de corante.

Os experimentos foram realizados em um agitador termostatizado (Innova 44, New Brunswick Scientific, EUA) a $25^{\circ} \mathrm{C}$, sob agitação de $150 \mathrm{rpm}$. Alíquotas foram retiradas em intervalos de tempo prédeterminados $(2,4,6,8,10,15,20,25,30$, $40,50,60,80,90,100,120,140,160$ e 180 min) (DOTTO; PINTO, 2011).

As alíquotas da solução foram filtradas (papel filtro Whatmann $\mathrm{n}^{\circ} 40$ ), e as concentrações dos corantes foram determinadas por espectrofotometria (Quimis, Q108, Brasil). Todos os experimentos foram realizados em triplicata e testes de branco foram realizados.

As concentrações finais $\left(\mathrm{mg} \quad \mathrm{L}^{-1}\right)$ corantes amarelo tartrazina $\left(\mathrm{C}_{\mathrm{A}}\right)$ e azul indigotina $\left(C_{B}\right)$ no sistema binário, foram calculadas de acordo com as Equações 1 e 2 (MAHMOODI et. al., 2011). 


$$
\begin{aligned}
& C_{A}=\frac{\left(k_{B 2} d_{1}-k_{B 1} d_{2}\right)}{\left(k_{A 1} k_{B 2}-k_{A 2} k_{B 1}\right)} \\
& C_{B}=\frac{\left(k_{A 1} d_{2}-k_{A 2} d_{1}\right)}{\left(k_{A 1} k_{B 2}-k_{A 2} k_{B 1}\right)}
\end{aligned}
$$

onde, $k_{A 1}, k_{B 1}, k_{A 2}$ e $k_{B 2}$ são as constantes de calibração para componentes $\mathrm{A}$ e $\mathrm{B}$ em dois comprimentos de onda $\lambda 1$ e $\lambda 2$, para o corante amarelo tartrazina e azul indigotina, respectivamente.

A capacidade de adsorção no tempo $t$ $\left(q_{t}\right) \quad\left(\mathrm{mg} \mathrm{g}^{-1}\right)$ para cada corante foi determinada de acordo com a Equação 3.

$$
q_{t}=\frac{\left(C_{0}-C_{t}\right)}{m} V
$$

onde, $C_{0}$ e $C_{t}$ são as concentrações inicial e no tempo $t$ na fase líquida $\left(\mathrm{mg} \mathrm{L}^{-1}\right), V$ o volume da solução (L), $m$ a massa de quitosana $(\mathrm{g})$.

\subsection{Modelos cinéticos}

Para avaliar o comportamento cinético de adsorção foram utilizados os modelos cinéticos de pseudo-primeira ordem, pseudosegunda ordem e Avrami.

Os modelos cinéticos de pseudo-primeira ordem e pseudo-segunda ordem assumem que a adsorção é uma reação pseudo-química. A capacidade de adsorção em função do tempo para cada modelo pode ser calculada de acordo com as Equações 4 e 5.

$$
q_{t}=q_{1}\left(1-\exp \left(-k_{1} t\right)\right)
$$

$q_{t}=\frac{t}{\left(1 / k_{2} q_{2}^{2}\right)+\left(t / q_{2}\right)}$

onde, $k_{1}$ e $k_{2}$ são os coeficientes cinéticos de pseudo-primeira e pseudo-segunda ordens $\left(\mathrm{min}^{-1}\right)$ e $\left(\mathrm{g} \mathrm{mg}^{-1} \mathrm{~min}^{-1}\right), q_{t}$ corresponde à quantidade de adsorbato adsorvido por unidade de massa de adsorvente no instante $t$, $q_{1}$ e $q_{2}$ são os valores teóricos para a capacidade de adsorção (mg $\mathrm{g}^{-1}$ ) obtidos através dos modelos de pseudo-primeira ordem e pseudo-segunda ordem, respectivamente.

O modelo de Avrami é baseado em uma cinética de decomposição térmica (LOPES et al., 2003). Está representado de acordo com a Equação 5.

$q_{t}=q_{A V}\left(1-\exp \left(-k_{A V} t\right)^{n}\right)$

onde, $k_{A V}$ é a constante cinética de Avrami $\left(\mathrm{min}^{-1}\right), \quad q_{A V}$ é a capacidade teórica de adsorção de Avrami $\left(\mathrm{mg} \mathrm{g}^{-1}\right)$ e $n$ é o expoente fracionário.

Os parâmetros das cinéticas foram determinados por regressão não-linear utilizando o método de estimação HookeJeeves (Statistica 7.0, Statsoft, EUA). A qualidade do ajuste foi verificada de acordo com o coeficiente de determinação $\left(\mathrm{R}^{2}\right)$ e o erro médio relativo (EMR). Os resultados foram avaliados estatisticamente através do Teste do Tukey ao nível de $95 \%$ de confiança $(\mathrm{p}<0,05)$.

\section{RESULTADOS E DISCUSSÃO}

\subsection{Caracterização da quitosana com e sem modificação}

As características texturais da quitosana com e sem modificação, foram observadas através da análise de microscopia eletrônica de varredura (MEV) que está apresentada na Figura 2

A partir da Figura 2(a) pode ser observado que a quitosana sem modificação apresentou-se mais homogênea, com superfície mais lisa e ausência de poros. Já a quitosana modificada (Figura 2(b)) retratou uma superfície com concavidades e 
protuberâncias, heterogênea e sem poros. Ambas as quitosana apresentaram um diâmetro do pó menor $75 \mu \mathrm{m}$.

Figura 2 - Imagens de MEV para (a) quitosana sem modificação (b) quitosana modificada

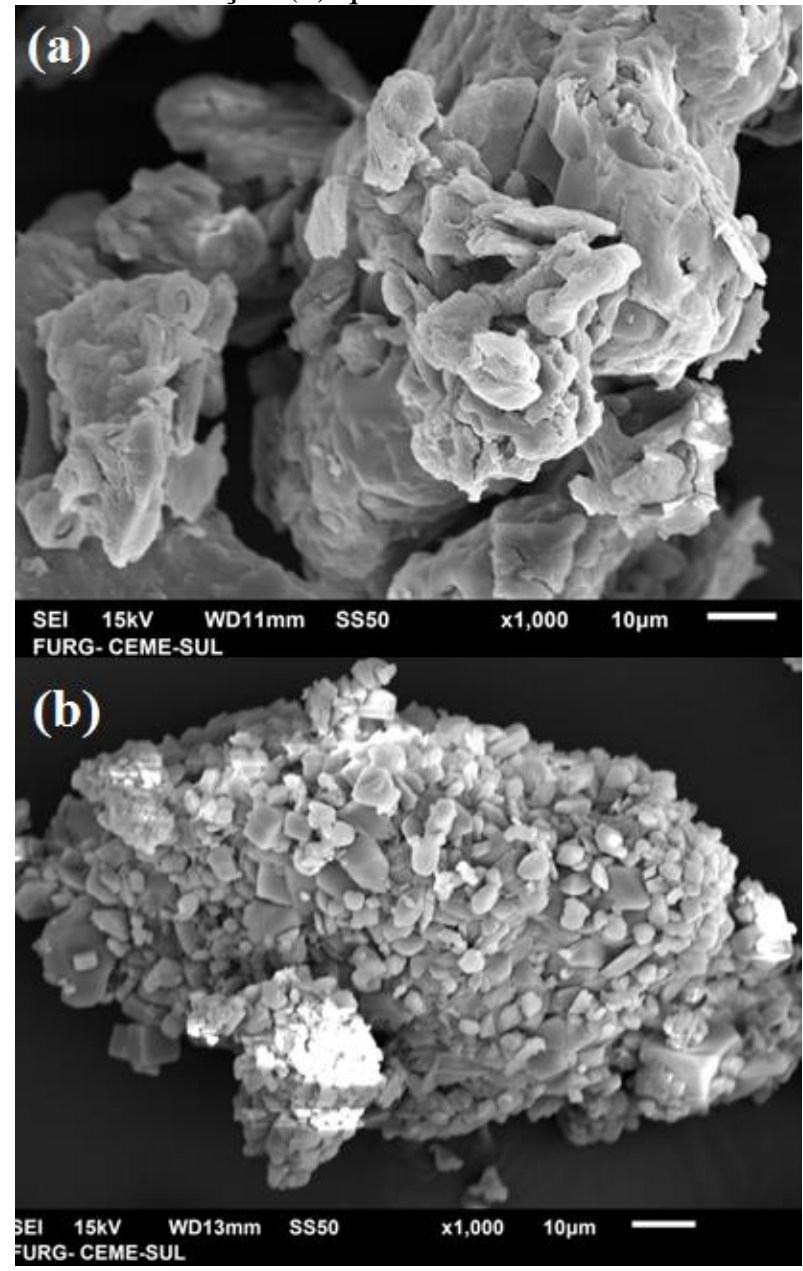

Fonte: AUTORES (2015)

Com o intuito de identificar alterações nos grupos funcionais nas diferentes amostras de adsorventes, foram realizadas análises por FT-IR, que está apresentada na Figura 3.

$\mathrm{Na}$ Figura 3(a) verificaram-se as bandas características do pó de quitosana. Os estiramentos das ligações $\mathrm{N}-\mathrm{H}$ e $\mathrm{O}-\mathrm{H}$ foram observados nos picos 3350 e $3150 \mathrm{~cm}^{-1}$, respectivamente. A vibração de $\mathrm{C}=\mathrm{O}$ (banda amida I) foi observada em $1650 \mathrm{~cm}^{-1}$. No pico $1550 \mathrm{~cm}^{-1}$, é observado o estiramento da ligação $\mathrm{C}-\mathrm{N}$ da amida. As deformações angulares de C-O-H e $\mathrm{H}-\mathrm{C}-\mathrm{H}$ aparecem em $1450 \mathrm{~cm}^{-1}$. Em $1000 \mathrm{~cm}^{-1}$ pode ser verificado o estiramento C-O (DOTTO et al., 2013). A deformação angular da ligação $\mathrm{N}-\mathrm{H}$ pode ser verificada em $680 \mathrm{~cm}^{-1}$ (CADAVAL JR. et. al., 2013).

Figura 3 - Espetro infravermelho (FT-IR) (a) quitosana sem modificação (b) quitosana com modificação
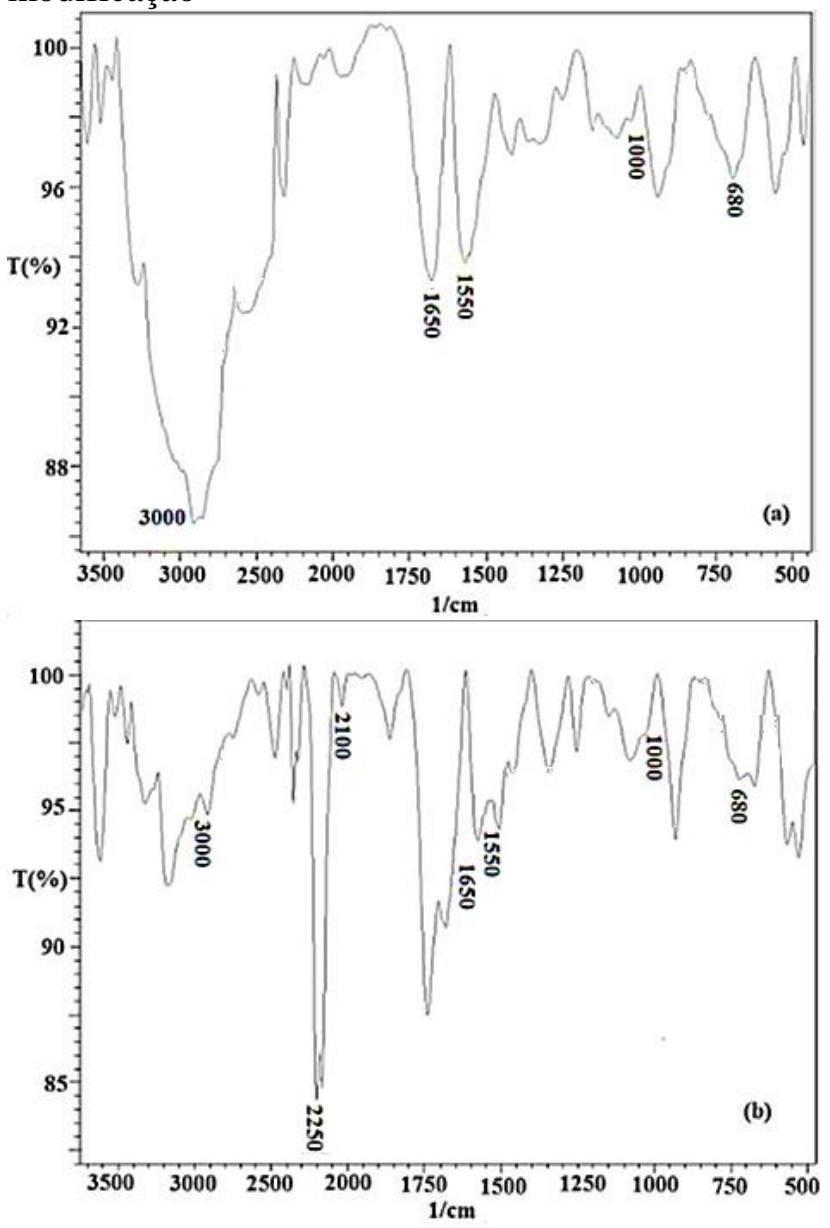

Fonte: AUTORES (2015)

Na Figura 3(b), a principal alteração que pode ser observada após a modificação da quitosana, foi uma banda intensa em torno da banda de $2250 \mathrm{~cm}^{-1}$, que é de um vínculo de sal de amônio formado (ZHAO et. al., 2012). Uma banda em $2100 \mathrm{~cm}^{-1}$ apareceu, referente a um pico característico da $\mathrm{C} \equiv \mathrm{N}$ da cianoguanidina. A banda larga em torno de 
$3000 \mathrm{~cm}^{-1}$ diminuiu devido à inserção da cianoguanidina no grupo amino de quitosana.

\subsection{Cinética de adsorção}

A Figura 4 apresenta as curvas cinéticas da adsorção dos corantes em sistema binário por quitosana com e sem modificação.

Figura 4 - Curvas cinéticas de adsorção para os corantes azul indigotina e amarelo tartrazina (a) quitosana sem modificação (d) quitosana com modificação
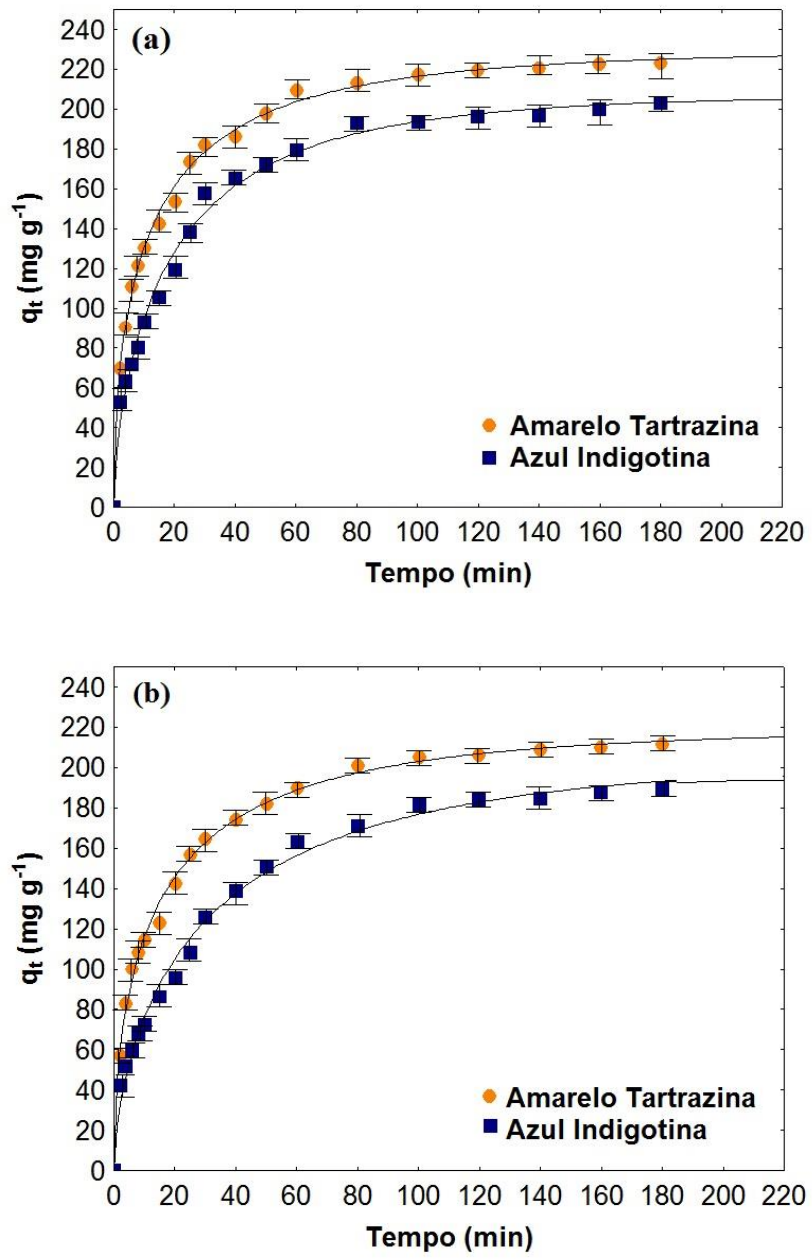

Fonte: Autores (2015)

$\mathrm{Na}$ Figura 4, verificou-se que a quitosana sem modificação (Figura 4(a)) obteve uma maior capacidade de adsorção de ambos os corantes do que a quitosana modificada (Figura 4(b)). Isto pode ter ocorrido devido à modificação ocupar alguns sítios reativos da quitosana, assim justificando a diminuição da capacidade de adsorção.

O corante amarelo tatrazina apresentou maiores valores da capacidade de adsorção do que o azul indigotina. Este comportamento pode ser verificado para ambos os adsorventes, mostrando a preferência da quitosana modificada pelo corante amarelo tartrazina. Este fato pode ser explicado devido à estrutura do corante e ao tamanho da molécula (Figura 1 e 2). O corante amarelo tartrazina apresenta dois grupos sulfonados e um grupo carboxila em sua estrutura, já o azul indigotina apresenta apenas dois grupos sulfonados. Portanto, o amarelo tartrazina tem mais grupos para interagir com $\mathrm{NH}_{3}$ e $\mathrm{OH}$ do adsorvente (DOTTO et al., 2015).

\subsection{Modelos cinéticos}

A Tabela 2 apresenta o ajuste dos dados experimentais cinéticos aos modelos de pseudoprimeira ordem, pseudo-segunda ordem e Avrami.

Na Tabela 2 verificou-se que o modelo mais adequado para representar a cinética de adsorção dos corantes por ambos os adsorventes foi o modelo de Avrami $\left(\mathrm{R}^{2}>0,95\right.$ e ERM $<10 \%$ ). 
Tabela 2 - Ajuste dos modelos cinéticos da adsorção de corantes por diferentes quitosanas.

\begin{tabular}{lcccc}
\hline \multirow{2}{*}{ Modelos } & \multicolumn{2}{c}{ Quitosana sem modificação } & \multicolumn{2}{c}{ Quitosana modificada } \\
\cline { 2 - 5 } & $\begin{array}{c}\text { Amarelo } \\
\text { tartrazina }\end{array}$ & $\begin{array}{c}\text { Azul } \\
\text { indigotina }\end{array}$ & $\begin{array}{c}\text { Amarelo } \\
\text { tartrazina }\end{array}$ & $\begin{array}{c}\text { Azul } \\
\text { indigotina }\end{array}$ \\
\hline PPO & & & & \\
$q_{1}\left(\mathrm{mg} \mathrm{g}^{-1}\right)$ & 208,7 & 192,7 & 196,5 & 181,8 \\
$k_{1}\left(\mathrm{~min}^{-1}\right)$ & 0,0975 & 0,0588 & 0,0845 & 0,0428 \\
$\mathrm{R}^{2}$ & 93,3 & 96,1 & 93,4 & 96,0 \\
ERM $(\%)$ & 10,45 & 7,30 & 11,54 & 9,21 \\
\hline PSO & & & & \\
$q_{2}\left(\mathrm{mg} \mathrm{g}^{-1}\right)$ & 228,4 & 216,0 & 216,4 & 209,0 \\
$k_{2}\left(\mathrm{~g} \mathrm{mg}^{-1} \mathrm{~min}^{-1}\right)$ & 0,0006 & 0,0003 & 0,0005 & 0,0002 \\
$\mathrm{R}^{2}$ & 98,5 & 98,3 & 98,4 & 98,1 \\
ERM $(\%)$ & 4,75 & 6,08 & 8,27 & 5,49 \\
\hline Avrami & & & & \\
$q_{a v}\left(\mathrm{mg} \mathrm{g}^{-1}\right)$ & 229,5 & 207,0 & 217,7 & 204,4 \\
$k_{A V}\left(\mathrm{~min}^{-1}\right)$ & 0,2433 & 0,1379 & 0,2156 & 0,1075 \\
$\mathrm{~N}$ & 0,5355 & 0,6509 & 0,5458 & 0,6347 \\
$\mathrm{R}^{2}$ & 99,7 & 99,2 & 99,7 & 99,3 \\
ERM $(\%)$ & 1,56 & 4,12 & 1,82 & 4,65 \\
\hline
\end{tabular}

PPO:pseudo-primeira ordem, PSO: pseudo-segunda ordem

Fonte: Autores (2015)

\section{CONCLUSÃO}

Os adsorventes foram obtidos e caracterizados. A quitosana apresentou uma superfície homogênea e lisa, e a quitosana modificada mostrou-se heterogênea, com concavidades e protuberâncias. Os adsorventes apresentaram diferentes grupamentos funcionais, onde foi observado a inserção da cianoguanidina na quitosana. As capacidades máximas de adsorção para os corantes foram de aproximadamente $225 \mathrm{mg}$ $\mathrm{g}^{-1}$ e $205 \mathrm{mg} \mathrm{g}^{-1}$ para o amarelo tartrazina e azul indigotina, respectivamente, sendo que os adsorventes não apresentaram diferença significativa $(\mathrm{p}<0,05)$. O modelo de Avrami foi o que melhor se ajustou aos dados experimentais.

\section{REFERÊNCIAS}

CADAVAL, JR. T. R. S.; CAMARA, A. S.; DOTTO, G. L.; PINTO, L. A. A. Adsorption of $\mathrm{Cr}$ (VI) by chitosan with different deacetylation degrees. Desalination and Water Treatment, v.51, p.7690-7699, 2013.

DEMIRBAS, A. Agricultural based activated carbons for the removal of dyes from aqueous solutions: A review. Journal of Hazardous Materials, v.167 p.1-9, 2009.

DOTTO, G. L.; PINTO, L. A. A.; HACHICHA, M. A.; KNANI, S. New physicochemical interpretations for the adsorption of food dyes on chitosan films using statistical physics treatment. Food Chemistry, v.171, p.1-7, 2015.

DOTTO, G. L.; MOURA, J. M.; CADAVAL, JR. T. R. S.; PINTOL. A. A. Application of 
chitosan films for the removal of food dyes from aqueous solutions by adsorption. Chemical Engineering Journal, v.214, p.816, 2013.

DOTTO, G. L.; PINTO, L. A. A. Adsorption of food dyes acid blue 9 and food yellow 3 onto chitosan: Stirring rate effect in kinetics and mechanism. Journal of Hazardous Materials, v.187, p.164-170, 2011.

DOTTO, G. L.; SOUZA, V. C.; PINTO, L. A. A. Drying of chitosan in a spouted bed: The influences of temperature and equipment geometry in powder quality. LWT -Food Science and Technology International, v. 44, p. 1786-1792, 2011.

DRONSKOWSKI, R.; HUI-LIU, X. Bis(cyanoguanidine) silver (I) Nitratecyanoguanidine. Acta Crystallographica Section C/Crystal Structure Communications, v. 59, p. 243-245, 2003.

FU, F.; WANG, Q. Removal of heavy metal ions from wastewaters: A review. Journal of Environmental Management, v.92, p.407418, 2011.

GUPTA, V. K.; SUHAS. Application of lowcost adsorbents for dye removal- A review. Journal of Environmental Management, v.90, p.313-2342, 2009.

KUNZ, A.; PERALTA-ZAMOTRA, P.; MORAES, S. G.; DURÁN, N. Novas tendências no tratamento de efluentes têxteis. Química Nova, v.25, p.78-82, 2002.

LOPES, E. C. N.; ANJOS, F. S. C.; VIEIRA, E. F. S.; CESTARI, A. R. An alternative Avrami equation to evaluate kinetic parameters of the interaction of $\mathrm{Hg}$ (II) with thin chitosan membranes. Journal of Colloid and Interface Science, v.263, p.542-547, 2003.
MAHMOODI, N. M.; SALEHI, R.; ARAMI, M.; BAHRAMI, H. Dye removal from colored textile wastewater using chitosan in binary systems. Desalination, v.267, p.64-72, 2011.

MOURA, C. M.; MOURA, J. M.; SOARES, N. M.; PINTO, L. A. A. Evaluation of molar weight and deacetylation degree of chitosan during chitin deacetylation reaction: Used to produce biofilm. Chemical Engineering and Processing, v.50, p.351-355, 2011.

RINAUDO, M. Chitin and chitosan: Properties and applications. Progress in Polymer Science, v.31, p. 603-632, 2006.

SALLEH, M. A. M.; MAHMOUD, D. K.; KARIM, W. A. W. A.; IDRIS, A. Cationic and anionic dye adsorption by agricultural solid wastes: A comprehensive review. Desalination, v.280 p.1-13, 2011.

SRINIVASAN, A.; VIRARAGHAVAN, T. Decolorization of dye wastewaters by biosorbents: A review. Journal of Environmental Management, v.91, p.19151929, 2010.

ZHAO, X.; QIAO, Z. Z.; HE, J. X. Preparation of chitosan biguanidine hydrochloride and application in antimicrobial finish of wool fabric. Journal of Engineering Fibers Fabrics, v.5, p.3-20, 2012.

ZHOU, L.; MENG, X.; FU J.; YANGA, Y. C.; YANGA, P.; MI C. Highly efficient adsorption of chlorophenols onto chemically modified chitosan. Applied Surface Science, v. 292, p. 735-741, 2014.

WESKA, R. F.; MOURA, J. M.; BATISTA, L. M.; RIZZI, J.; PINTO, L. A. A. Optimization of deacetylation in the 
production of chitosan from shrimp wastes: Use of response surface methodology. Journal of Food Engineering, v. 80, p. 749753, 2007.

WAN NGAH, W. S.; TEONG, L. C.; HANAFIAH, M. A. K. M. Adsorption of dyes and heavy metal ions by chitosan composites: A review. Carbohydrate Polymer. v.83, p.1446-1456, 2011.

WANG, Y.; QI, Y.; LI, Y.; WU, J.; MA, X.; YU, C.; JI L. Preparation and characterization of a novel nano-absorbent based on multicyanoguanidine modified magnetic chitosan and its highly effective recovery for $\mathrm{Hg}$ (II) in aqueous phase. Journal of Hazardous Materials, v.260, p.9-15, 2013.

\section{AGRADECIMENTOS}

Os autores agradecem a CAPES, CNPq, FAPERGS, FURG pelo auxílio financeiro, e também ao CEME-SUL/FURG (Centro de Microscopia Eletrônica da Metade Sul) pelo suporte analítico. 